\title{
Systematic Review and Meta-analysis of Efficacy and Safety of Melatonin and Triclofos for Inducing Adequate Sedation for Sleep Electroencephalogram in Children
}

\author{
Prateek K. Panda ${ }^{1}$ Pragnya Panda ${ }^{2}$ Lesa Dawman ${ }^{3}$ Indar K. Sharawat ${ }^{1}$ (0) \\ ${ }^{1}$ Division of Pediatric Neurology, Department of Pediatrics, All India \\ Institute of Medical Sciences, Rishikesh, Uttarakhand, India \\ 2 Department of Neurology, King George's Medical University, \\ Lucknow, Uttar Pradesh, India \\ Address for correspondence Indar K. Sharawat, DM, Division of \\ Pediatric Neurology, Department of Pediatrics, All India Institute of \\ Medical Sciences, Rishikesh, Uttarakhand, India 249203 \\ (e-mail: sherawatdrindar@gmail.com).
}

${ }^{3}$ Department of Pediatrics, Post Graduate Institute of Medical Education and Research, Chandigarh, India

J Neurosci Rural Pract 2022;13:3-11.

\begin{abstract}
Keywords

- electroencephalography

- triclofos

- melatonin

- sedation

- EEG
\end{abstract}

Introduction Triclofos and melatonin are commonly used oral sedatives in children for obtaining a sleep electroencephalogram (EEG) record. There has been no systematic review till now to compare the efficacy and safety of these two medications.

Objectives The review intended to compare the efficacy of oral triclofos and melatonin in children $<18$ years of age for inducing adequate sedation for obtaining a sleep EEG record. We also attempted to compare the adverse effects, impact on EEG record, the yield of epileptiform abnormalities, and sleep onset latency in both groups. Methods A systematic search was conducted on "MEDLINE/PUBMED, Cochrane Central Register of Controlled Trials (CENTRAL), EMBASE, Web of Science, and Google Scholar" till November 30, 2020, with the following keywords/the Medical Subject Headings (MESH) terms while searching: "sleep EEG," "electroencephalogram," "triclofos," “melatonin" OR "ramelteon" AND “epilepsy," "seizure," OR "convulsion." ROB 2.0 and ROBINS-I tool was used to determine the risk of bias. To assess heterogeneity in studies, Higgins and Thompson's $I^{2}$ method was utilized. When $I^{2}$ was more than $50 \%$, a random effects model was utilized and a fixed-effect model was used for other parameters. To assess the presence of publication bias, Egger's test was used.

Results For describing the efficacy of triclofos in 1,284 and melatonin in 1,532 children, we selected 16 articles. The indirect comparison between the pooled estimate of all children receiving individual medications revealed comparable efficacy in obtaining successful sleep EEG record with a single dose (90 vs. $76 \%, p=0.058$ ) and repeat dose $(p=0.054)$, detection of epileptiform abnormalities $(p=0.06)$, and sleep onset latency $(p=0.06)$, but more proportion of children receiving triclofos had adverse effects $(p=0.001)$ and duration of sleep was also higher with triclofos $(p=0.001)$. published online December 30, 2021
DOI https://doi.org/

10.1055/s-0041-1736511. ISSN 0976-3147. (c) 2021. Association for Helping Neurosurgical Sick People. All rights reserved.

This is an open access article published by Thieme under the terms of the Creative Commons Attribution-NonDerivative-NonCommercial-License, permitting copying and reproduction so long as the original work is given appropriate credit. Contents may not be used for commercial purposes, or adapted, remixed, transformed or built upon. (https://creativecommons.org/ licenses/by-nc-nd/4.0/)

Thieme Medical and Scientific Publishers Pvt. Ltd., A-12, 2nd Floor, Sector 2, Noida-201301 UP, India 
Conclusion Efficacy of triclofos and melatonin are comparable in inducing sleep for recording EEG in children, although triclofos is more likely to cause adverse effects. However, the level of evidence is low for this conclusion and the weak strength of recommendation for the results of this review is likely to change in the future after completion of controlled trials exploring these two medications.

\section{Introduction}

Young children often do not cooperate for recording a highquality sleep electroencephalogram (EEG) record, especially in the subgroup with developmental delay or behavioral problems. Moreover, even if awake EEG remains feasible for some of these children, for most epilepsies, the yield of epileptiform abnormalities remains higher with non-rapid eye movement (NREM) sleep EEG records. ${ }^{1}$ Thus, often oral sedatives are used in children and sometimes even in adults for inducing sleep to facilitate EEG recording. The pineal gland secrets the hormone melatonin which plays a key role in maintaining the sleep-wake cycle. ${ }^{2}$ Its efficacy and safety for inducing sleep for various noninvasive procedures in adults, children, and even in those with developmental or behavioral impairment are well known. ${ }^{3}$ Moreover, being an indigenous product of the human body, its acceptance as a sedative in children has increased many folds over the last decade. Similarly, triclofos, which is a chloral hydrate analogue, is also in use for many years now and its efficacy and safety have also been proven in many studies. ${ }^{4}$ Chloral hydrate, chlorpromazine, and promethazine were previously used for inducing sleep for neurophysiological procedures like EEG. But over time, their popularity has been reduced due to unacceptable adverse effect profiles of these drugs like prolonged sedation and even respiratory depression, hypotension, and desaturation in few cases. ${ }^{5}$ For such purposes, the Food and Drug Administration (FDA) has issued warnings over the use of promethazine in children $<2$ years. ${ }^{6}$ While the use of intravenous or oral midazolam for sleep EEG recording remains controversial, it has the probability of affecting the yield of epileptiform abnormalities. ${ }^{7}$ Although such a claim has never been proven definitely in clinical studies, its acceptance remains limited among clinicians for this purpose. Thus, many clinicians use triclofos and melatonin for inducing sleep for EEG recording. Although the choice of sedative depends on the clinician and till date, there is no definite protocol to choose which sedative initially and which one after the failure of initial sedative; in developing countries, especially, the above-mentioned two drugs are being used abundantly.

\section{Methods}

\section{Search Methods}

The systematic review was performed to compare the evidence on efficacy, safety, tolerability/adverse effects, and impact on the yield of detection of epileptiform discharges, as well as the prevalence of sedative-related artifacts in children receiving triclofos and melatonin for obtaining sleep EEG record from the currently available literature. The primary objective of the review was to compare the efficacy of oral triclofos and melatonin in achieving adequate sedation for obtaining successful sleep EEG records in children aged less than 18 years with/without any developmental delay/behavioral problems. Secondary objectives were to compare the efficacy of these two medications in attaining sedation in the subgroup of children with developmental or behavioral problems, yield of epileptiform abnormalities, sleep onset latency, duration of adequate sleep, proportion requiring the second dose of sedative, proportion of children in whom the procedure was differed because of the ineffectiveness of each of the sedative, and proportion of EEG records with sedative-related artifacts in both groups. We also tried to determine whether there was any difference between the efficacy and safety of selective melatonin receptor agonist, ramelteon, and triclofos as another secondary objective.

The safety-related secondary objectives were to determine the prevalence and nature of various adverse effects with each of these two sedatives and proportion of patients with serious adverse effects/death. The predominant adverse effects, the review concentrated on, were excessive sedation/drowsiness; agitation, irritability; gastrointestinal side effects like vomiting, gastric irritation, and diarrhea; cardiovascular adverse effects like hypotension and bradycardia; and respiratory adverse effects like desaturation, respiratory depression, and others.

While performing the systematic review and the metaanalysis and reporting the results, the following statements/recommendations were adhered with: "the PRISMA (Recommendations of the Preferred Reporting Items for Systematic Reviews and Meta-Analyses) and the MOOSE (meta-analysis of observational studies in epidemiology)."

Before commencing the review process, a predefined search strategy was developed. A systematic literature search (on December 1,2020 ) for all articles published till November 30, 2020, on the following databases: "MEDLINE/PUBMED, Cochrane Central Register of Controlled Trials (CENTRAL), EMBASE, Web of Science, and Google Scholar" was independently performed by two authors (P.K.P. and I.K.S.). While searching the literature, the following keywords/MESH terms were used: "sleep EEG," "electroencephalogram," "triclofos," "melatonin," and "ramelteon." The MESH terms, like "epilepsy," "seizure," and "convulsion," were used in combination with these terms. As described below, after initial scrutiny, to identify additional studies, relevant search items were 
selected and the references of these search items and pertinent review articles were screened.

\section{Eligibility of Studies}

We included only original articles describing studies with both prospective/retrospective study designs, either enrolling children receiving triclofos and/or melatonin/ ramelteon. As we could not get many studies directly comparing the efficacy of triclofos and melatonin in children for inducing sleep, we attempted to perform an indirect comparison by comparing the pooled estimate of the efficacy of individual medications. To obtain a true pooled estimate, only studies with a sample size of 30 or more receiving any of the above two medications/both medications were included.

The studies with incomplete details were excluded from corresponding quantitative review to avoid bias and obtain a true pooled estimate. The studies without a well-defined measure of sleep intensity/attainment of sleep, which is the primary endpoint of the review, were also excluded.

\section{Study Selection, Data Extraction, and Assessment of the Risk of Bias}

After initial scrutiny of title, abstract, and study design, two independent reviewers performed the full-text review for all the selected search items included. Based on the consensus opinion of both reviewers after applying the above-mentioned inclusion and exclusion criteria, the final set of studies were selected to be included in the quantitative review/ meta-analysis. To resolve the dispute, in case any dispute occurred between both reviewers, the opinion of a third reviewer was taken. The included studies subsequently underwent assessment of the methodological quality, using validated quality assessment tools. From the included articles, relevant data were extracted after full-text review depending on the study methodology and outcome variables, that is, study design, study period, number and characteristics of the sample population, their demographic and epilepsy variables, comedications (antiseizure medications [ASMs] and psychotropic drugs), developmental/behavioral comorbidities, whether they were advised partial sleep deprivation the night before, the proportion with successful sleep EEG recording with single/multiple doses of the medication, yield of epileptiform abnormalities, proportion of sleep EEGs with sedation-related artifact, duration of sleep, sleep onset latency, nature, incidence of adverse effects and serious adverse effects, dose of the medications, and others.

The data were transferred to a Microsoft Excel spreadsheet after uniform and systematical extraction of data in a standardized predetermined form. By mutual discussion, the review was resolved for any discrepancies regarding inclusion. To ensure the accuracy and completeness of the extracted data, another third independent investigator was asked to perform quality check for the extracted data. If some disagreement used to occur between both investigators on some topic, then the third investigator was involved in the discussion to achieve a consensus decision. To avoid duplication of data, all possible efforts were made. The cases, which were not included previously as part of another series only, were included in the final analysis.

The Newcastle Ottawa scale was used for the quality check of uncontrolled studies. Based on the scale, the studies were classified as good, fair, and poor quality. The Cochrane Collaboration's tool for assessing the risk of bias in randomized controlled trials (RCTs) or the Risk Of Bias In Nonrandomized Studies of Interventions (ROBINS-I) tool was used for the risk of bias assessment. ${ }^{8}$

\section{Outcome Measures}

The percentage of patients with successful sleep EEG recording with the first dose of triclofos and melatonin was taken as the primary efficacy outcome measure. The other efficacy outcomes were the proportion of children with developmental/behavioral problems in whom sleep EEG could be recorded, depth of sedation achieved with both these medications, proportion with successful sleep EEG after repeat dose of these medications, the yield of epileptiform abnormalities, percentage of children with artifacts in sleep EEG related to sedatives, and their grading and sleep onset latency. Outcomes related to safety and tolerability were the proportion of children with adverse effects, serious adverse effects, and deaths related to both these medications and the nature of these adverse effects.

\section{Data Synthesis and Statistical Analysis}

The categorical variables were expressed in frequency (in percentage) along with $95 \%$ confidence interval (CI) and the continuous variables were expressed as mean with standard deviation or median with interquartile range. Among the included variables in the review, the pooled estimate was determined along with upper and lower 95\% CIs, whenever it seemed feasible. RevMan 5.4 software and SPSS statistical software package were used to compare the pooled estimate of these parameters, including a meta-analysis of data regarding various parameters. To assess heterogeneity in studies, the Higgins and Thompson's $I^{2}$ method and Cochran's Q statistics with Chi-square test were utilized. The presence of publication bias was assessed using Egger's test. When $I^{2}$ was more than $50 \%$, a random effects model was utilized and a fixed-effect model was utilized for the rest of the parameters.

\section{Results}

\section{Results of the Search}

A total of 203 search items were retrieved after the initial targeted search. Ninety-one articles were excluded as they were found to be in duplicates. The remaining 112 articles were included in the initial evaluation for eligibility. Due to the irrelevance of the articles, based on either the title, abstract, or type of article, 79 articles were removed (-Fig. 1). Review for full text was performed for 33 articles and only 16 articles met the inclusion criteria for the review (4 studies describing the efficacy of triclofos, while 11 studies explored the efficacy of melatonin, and one retrospective study compared the efficacy of selective melatonin 


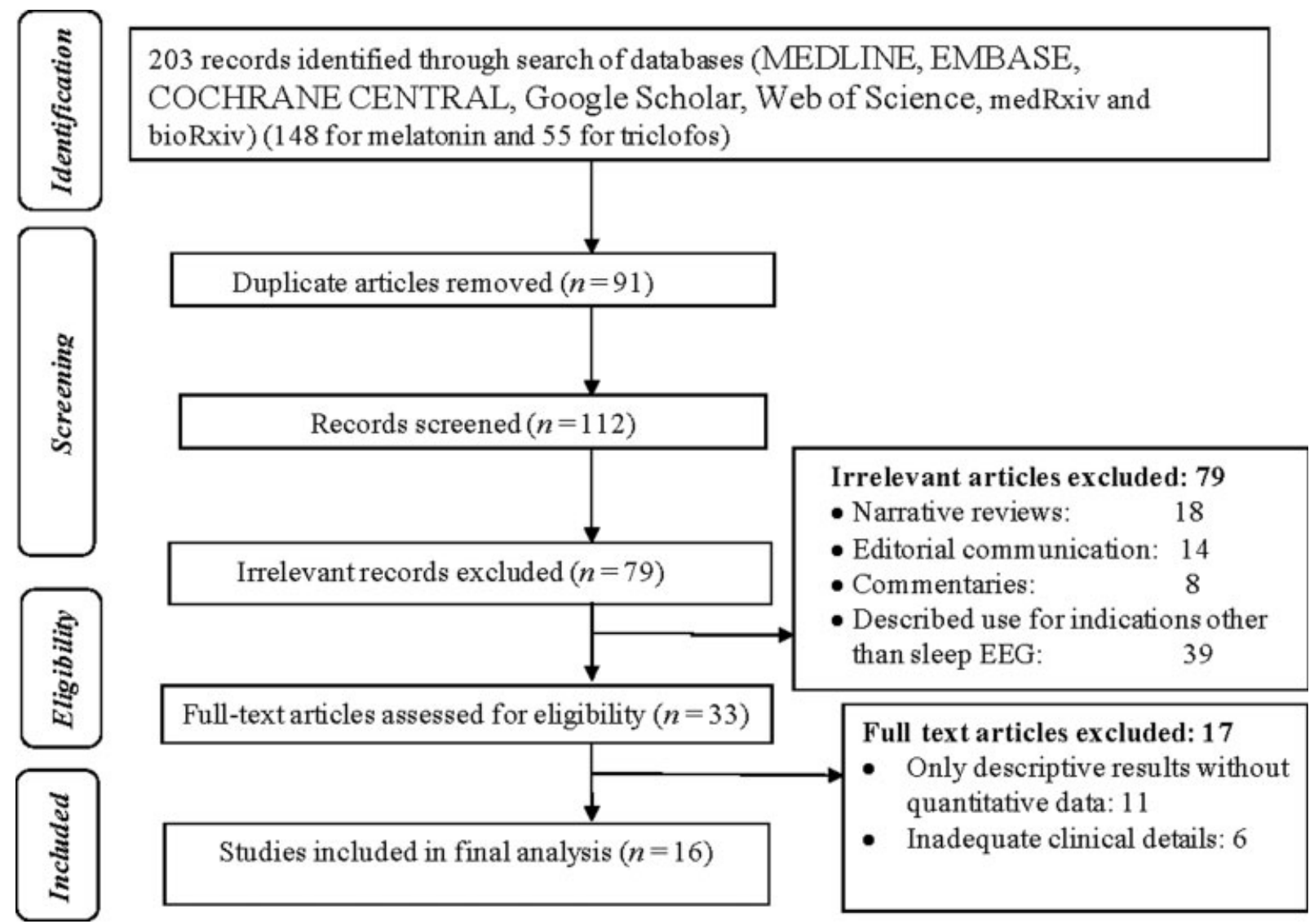

Fig. 1 Flow diagram of the study selection process. EEG, electroencephalogram.

receptor agonist, ramelteon, triclofos, and chloral hydrate). ${ }^{3,5,7,9-21}$

Out of the five studies exploring triclofos for sleep EEG, only one was a two-arm RCT in which efficacy, safety, and palatability of triclofos and chloral hydrate were compared and one was a prospective observational study. Rest of the three were retrospective studies ( - Supplementary Table S1; available in the online version). Similarly, out of the 12 studies describing the efficacy of melatonin, 3 studies each were RCT, nonrandomized controlled trial, prospective studies without comparison arm, and retrospective studies. While the six studies compared the efficacy of melatonin with partial sleep deprivation alone or in combination, two studies compared the efficacy of melatonin with chloral hydrate, one study with hydroxyzine and oral midazolam each (-Supplementary Table S2; available in the online version). As described above, only one study compared the efficacy of melatonin receptor agonist, ramelteon, and triclofos directly.

\section{Characteristics and Risk of Bias of Included Studies}

To assess the quality of the studies included, five studies were evaluated and only two were of good quality with low risk of bias and the rest were of fair quality. To explore the efficacy of melatonin, 12 studies were assessed and we found that 9 were of good quality and 3 were of fair quality. In 4 of the 16 studies, the risk of bias was low and in the rest of the studies, the bias was moderate according to Cochrane's collaboration tool and ROBINS-I tool. There were no poor- quality studies among those included in our review and no significant publication bias was noted.

We could not get any study directly comparing the efficacy and safety of triclofos and melatonin, apart from one retrospective study comparing ramelteon, melatonin receptor agonist, and triclofos. One RCT we could detect from Clinical Trials Registry- India (CTRI) is currently undergoing and comparing directly the efficacy of triclofos and melatonin in India, but the results are yet to be published, and hence it was excluded from the review. ${ }^{22}$ Hence, we attempted to perform an indirect comparison by collecting pooled estimates of all studies describing the efficacy and safety of these medications individually. The one study comparing directly ramelteon and triclofos included 23 children. In the triclofos group, the five studies included a total of 1,284 children and in the melatonin group, 12 studies included a total of 1,532 children. The age and gender distributions, as well as other demographic characteristics of the children included in both groups, were comparable, as well as the proportion of children on comedications like ASMs and psychotropic medications ( $p=0.480 .62$, respectively). Similarly, the proportion of children with developmental delay and behavioral abnormalities was also comparable between both groups $(p=0.31)$. Most of the children with developmental and behavioral problems had cerebral palsy, autism, or attention deficit hyperactivity disorder (ADHD). The epilepsy characteristics, like percentage of children who were already on $\operatorname{ASM}(p=0.31)$, number 
of ASMs, percentage of patients who previously had inconclusive or normal awake EEG, and duration of epilepsy, were also comparable between two groups ( $p=0.46,0.59$, and 0.68 , respectively for these variables). The main indications for sleep EEG were epilepsy or unprovoked seizure, unexplained behavioral abnormalities, learning, or cognition problems. The dose of melatonin used was 2.5 to $20 \mathrm{mg}$ (median: $5 \mathrm{mg}$ ) and the median dose of triclofos used was $50 \mathrm{mg} / \mathrm{kg} /$ day in these studies. The second dose was administered after an interval of 30 minutes to 1 hour if the first dose was found to be unsuccessful in these studies and subsequently either other sedatives were tried or the procedure differed. The median duration of sleep EEG recorded was 30 minutes in both groups, but it was mentioned only in a few studies in both groups.

Adequate sedation required for obtaining a satisfactory sleep EEG record was considered as the Ramsay sedation score 4 in most of the included studies. Percentage with such adequate sedation and successful EEG was comparable in both groups (90\% in triclofos arm vs. $76 \%$ in melatonin arm, $p=0.058$ ). After administration of the second dose, successful sedation could be achieved in an additional 5 and $6 \%$ of patients ( $p=0.74$; - Table 1 ). In 12 and $22 \%$, adequate sedation could not be achieved and the procedure has to be differed. Only a few studies mentioned the depth of sedation and the median Ramsay sedation score achieved was 4 in both groups. Sleep onset latency was also comparable between both groups and the successful sedation rate among the participating children with developmental/behavioral problems was also comparable $(p=0.06$ and 0.25 , respectively). Among the children, in whom successful sleep EEG was obtained, the percentage with epileptiform abnormalities detected in EEG, focal/diffuse slowing in EEG, and sedative related artifacts/diffuse fast activity in EEG were comparable between the two groups ( $p=0.06,0.92$, and 0.78 , respectively). However, duration of sleep was higher with triclofos $(p=0.001)$, the percentage of children with adverse effects, percentage of children with excessive sleepiness/drowsiness was also higher with triclofos ( $p=0.001$ and 0.01 , respectively; - Table 1 ). While none of the children receiving melatonin suffered from any serious adverse effects, in the triclofos group, five children had serious adverse effects; however, there was no significant statistical difference noted between the two groups in this regard $(p=0.11)$. The adverse effects of melatonin were mainly vomiting and irritation, whereas predominant adverse effects with triclofos were excessive sedation, rarely respiratory depression, apnea, bradycardia, and hypotension. However, in all of these cases, the clinical improvement occurred spontaneously and did not require inotropes or

Table 1 Comparison of variables describing pooled estimate of baseline characteristics, efficacy, and adverse effects of children receiving triclofos and melatonin for sleep EEG

\begin{tabular}{|c|c|c|c|}
\hline Variable & $\begin{array}{l}\text { Children receiving } \\
\text { triclofos }(n=1,284)\end{array}$ & $\begin{array}{l}\text { Children receiving } \\
\text { melatonin } \\
(n=1,532)\end{array}$ & p-Value \\
\hline $\begin{array}{l}\text { Age }(\mathrm{mo}) \\
\text { Mean }(\mathrm{SD})\end{array}$ & $36.83 \pm 15.28$ & $39.74 \pm 21.29$ & 0.07 \\
\hline Males (\%) & 61 & 63 & 0.88 \\
\hline Females (\%) & 39 & 37 & \\
\hline Proportion with developmental delay/behavioral abnormalities (\%) & 51 & 59 & 0.31 \\
\hline Proportion already on ASMs (\%) & 49 & 48 & 0.91 \\
\hline Proportion with adequate sedation and successful EEG recording (\%) & 90 & 76 & 0.054 \\
\hline Proportion with requirement of second dose (\%) & 9.8 & 19.2 & 0.10 \\
\hline $\begin{array}{l}\text { Proportion with adequate sedation and successful EEG recording } \\
\text { in children with developmental/behavioral problems (\%) }\end{array}$ & 79 & 71 & 0.25 \\
\hline Proportion with epileptiform abnormalities in EEG (\%) & 31.8 & 46 & 0.06 \\
\hline Proportion with focal or diffuse slowing in EEG (\%) & 6 & 7 & 0.92 \\
\hline Proportion with significant medication related artifacts in EEG (\%) & 7 & 9 & 0.78 \\
\hline Proportion with uninterpretable EEG tracing due to artifacts (\%) & 1 & 2 & 0.93 \\
\hline $\begin{array}{l}\text { Sleep onset latency (min) } \\
\text { Mean (SD) }\end{array}$ & $34.3 \pm 6.1$ & $24.2 \pm 7.3$ & 0.06 \\
\hline $\begin{array}{l}\text { Sleep duration (min) } \\
\text { Mean (SD) }\end{array}$ & $68.2 \pm 19.4$ & $25.4 \pm 6.2$ & 0.001 \\
\hline $\begin{array}{l}\text { Proportion with adverse effects (\%) } \\
\text { Mean (SD) }\end{array}$ & 16.8 & 1.27 & 0.001 \\
\hline $\begin{array}{l}\text { Proportion with excessive drowsiness/prolonged sleep duration (\%) } \\
\text { Mean (SD) }\end{array}$ & 11.5 & 0 & 0.01 \\
\hline
\end{tabular}

Abbreviations: EEG, electroencephalogram; SD, standard deviation. 
8 Melatonin and Triclofos for Sleep EEG Panda et al.

\begin{tabular}{|l|c|c|c|c|}
\hline Study & Sample size & Response rate (\%) & Cl lower (\%) & Cl upper (\%) \\
\hline Ashrafiet al, 2009 & 174 & 88.50575 & 74.52703 & 102.4845 \\
\hline Fallah et al, 2014 & 30 & 73.33333 & 42.68928 & 103.9774 \\
\hline Alix et al, 2019 & 238 & 72.26891 & 61.46842 & 83.06939 \\
\hline Sander et al, 2011 & 50 & 88 & 61.99766 & 114.0023 \\
\hline Dirani et al, 2016 & 213 & 44.60094 & 35.63206 & 53.56982 \\
\hline & & & & \\
\hline Eiserman et al, 2010 & 70 & 80 & 59.04672 & 100.9533 \\
\hline & & & & \\
\hline Gustafson et al, 2014 & 129 & 69.76744 & 55.35334 & 84.18154 \\
\hline & & & & \\
Wassmer et al, 2001 & 64 & 87.5 & 64.58235 & 110.4177 \\
\hline Wassmer et al, 2000 & 163 & 79.1411 & 65.48385 & 92.79836 \\
\hline Akaike et al, 2016 & 63 & 95.2381 & 71.13953 & 119.3367 \\
\hline & & & & \\
\hline Rizk et al, 2017 & 117 & 90.59829 & 73.35091 & 107.8457 \\
\hline Ibekwe et al, 2017 & 173 & 83.81503 & 70.17253 & 97.45753 \\
\hline Pooled estimate & 1532 & 76.37076 & 67.50568 & 85.23584 \\
\hline
\end{tabular}

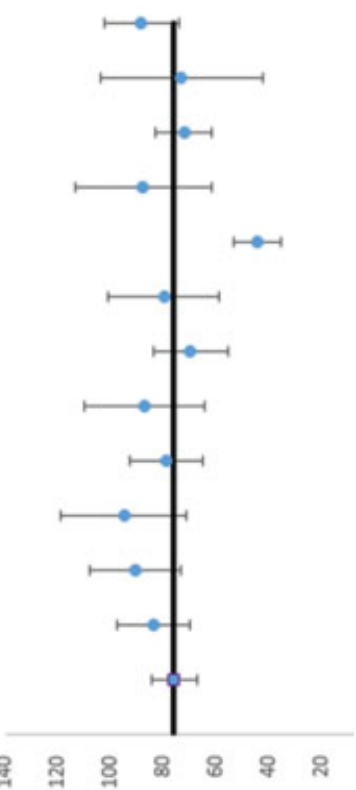

Heterogeneity: $\mathrm{Chi}^{2}=18.34, \mathrm{df}=11(p=0.07), I^{2}=42 \%$

Fig. 2 Meta-analysis forest plot showing pooled estimate for the percentage of children who had a successful sleep EEG record after the first dose of melatonin. EEG, electroencephalogram.

airway manipulation. No deaths have been reported with any of these drugs while being reported for this purpose. For inducing sleep in the evening and in young children, melatonin has been documented to be more helpful. Young age and prematurity were associated with respiratory complications with triclofos in one study, while the developmental delay was associated with hypotension in that study.

Efficacy $(p=0.64)$ and safety $(p=0.89)$ of ramelteon were found to be comparable with melatonin and even after excluding the study with ramelteon from the review, the comparison results remained similar. The efficacy for inducing sleep was slightly lower in children with developmental or behavioral impairment but no significant statistical difference was found for any of the medications $(p=0.09$ and 0.64 for triclofos and melatonin, respectively). Children with hypoxic-ischemic encephalopathy (HIE) and behavior abnormalities more frequently required second dose and rescheduling $(p=0.02)$.

Forest plots for the percentage of children with adequate sedation and successful EEG, the percentage with adverse effects, epileptiform abnormalities, mean sleep onset latency, and sleep duration in both groups have been demonstrated in -Figs. 2-4 and -Supplementary Figs. S1-S7 (available in the online version). For primary outcome variables and most of the secondary outcome variables, there was no significant heterogeneity. For any of these variables, no significant publication bias was observed.

\section{Discussion}

The results of this systematic review suggest that the efficacy of melatonin and triclofos as a sedative for facilitating sleep EEG was comparable in children, but melatonin had fewer adverse effects, making it a more reasonable initial choice in children. However, numerically describing the efficacy of triclofos appears to be higher, with the less-frequent requirement of the second dose, longer sleep duration. The prevalence of sedative-induced artifacts was numerically higher with triclofos, although the difference was insignificant.

Previously, several studies have suggested that melatonin has better efficacy in successfully facilitating sleep EEG as compared with sleep deprivation, while combined melatonin and sleep deprivation was found to be better than any of the individual interventions. ${ }^{13,14}$ The sleep duration obtained with melatonin is shorter, but in most cases, short-term video EEG records last up to 30 minutes which can be achieved with oral melatonin. Still, as compared with chloral hydrate, more children required the second dose but even the second dose did not lead to many adverse effects. The dose of melatonin seemed to vary more in the included studies as compared with a relatively constant value of the dose of triclofos. Thus, clinicians need to keep this in mind while using melatonin for sedation in children. Most of the studies used doses varying between 5 and $20 \mathrm{mg} /$ day, but even those receiving a higher dose or repeat doses had minimal or no adverse effects, suggesting an excellent safety profile of melatonin. ${ }^{9,10,13}$

Triclofos is a prodrug metabolized in the liver into the active form, trichloroethanol, which is considered pharmacologically as an active metabolite of chloral hydrate. ${ }^{18}$ Triclofos sodium of $1 \mathrm{~g}$ is roughly equivalent to $600 \mathrm{mg}$ of chloral hydrate. Triclofos causes less gastric irritation and has better palatability as compared with chloral hydrate, as shown in the study by Millichap and thus in recent years, it is considered as a better alternative for sedation, although the efficacy and adverse effect profile of both these 


\begin{tabular}{|l|c|c|c|c|}
\hline Study & Sample size & $\begin{array}{c}\text { Mean sleep onset } \\
\text { latency (in min) }\end{array}$ & Cl lower (in min) & Cl upper (in min) \\
\hline Ashrafi et al, 2009 & 174 & 45.0 & 23.722 & 66.278 \\
\hline Fallah et al, 2014 & 30 & 24.1 & 5.284 & 42.916 \\
\hline Alix et al, 2019 & 238 & 19.2 & 10.77 & 27.63 \\
\hline Sander et al, 2011 & 50 & 5.2 & 2.011 & 8.389 \\
\hline Dirani et al, 2016 & 213 & 31.3 & 16.44 & 46.16 \\
\hline Eiserman et al, 2010 & 70 & 25.0 & 9.66 & 40.33 \\
\hline Wassmer et al, 2001 & 64 & 23.1 & 19.964 & 26.236 \\
\hline wassmer et al, 2000 & 163 & 33.2 & 25.814 & 40.586 \\
\hline Akaike et al, 2016 & 63 & 22.3 & 9.473 & 35.127 \\
\hline Ibekwe et al, 2017 & 173 & 44.5 & 21.215 & 67.785 \\
\hline Pooled estimate & 1238 & 24.26 & 10.421 & 38.099 \\
\hline
\end{tabular}

Heterogeneity: $\mathrm{Chi}^{2}=14.93, \mathrm{df}=9(p=0.14), l^{2}=34 \%$

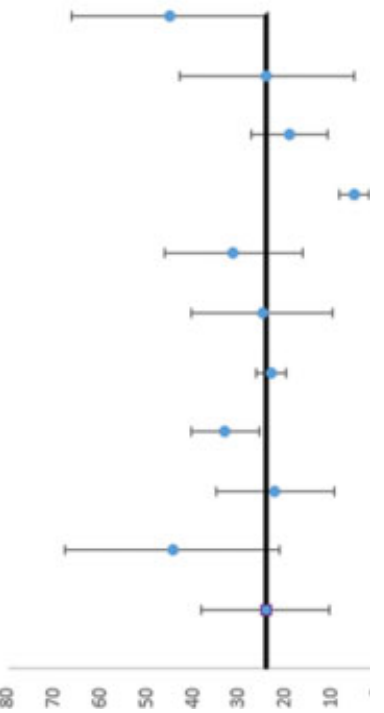

Fig. 3 Meta-analysis forest plot showing pooled estimate for sleep onset latency in children after the first dose of melatonin.

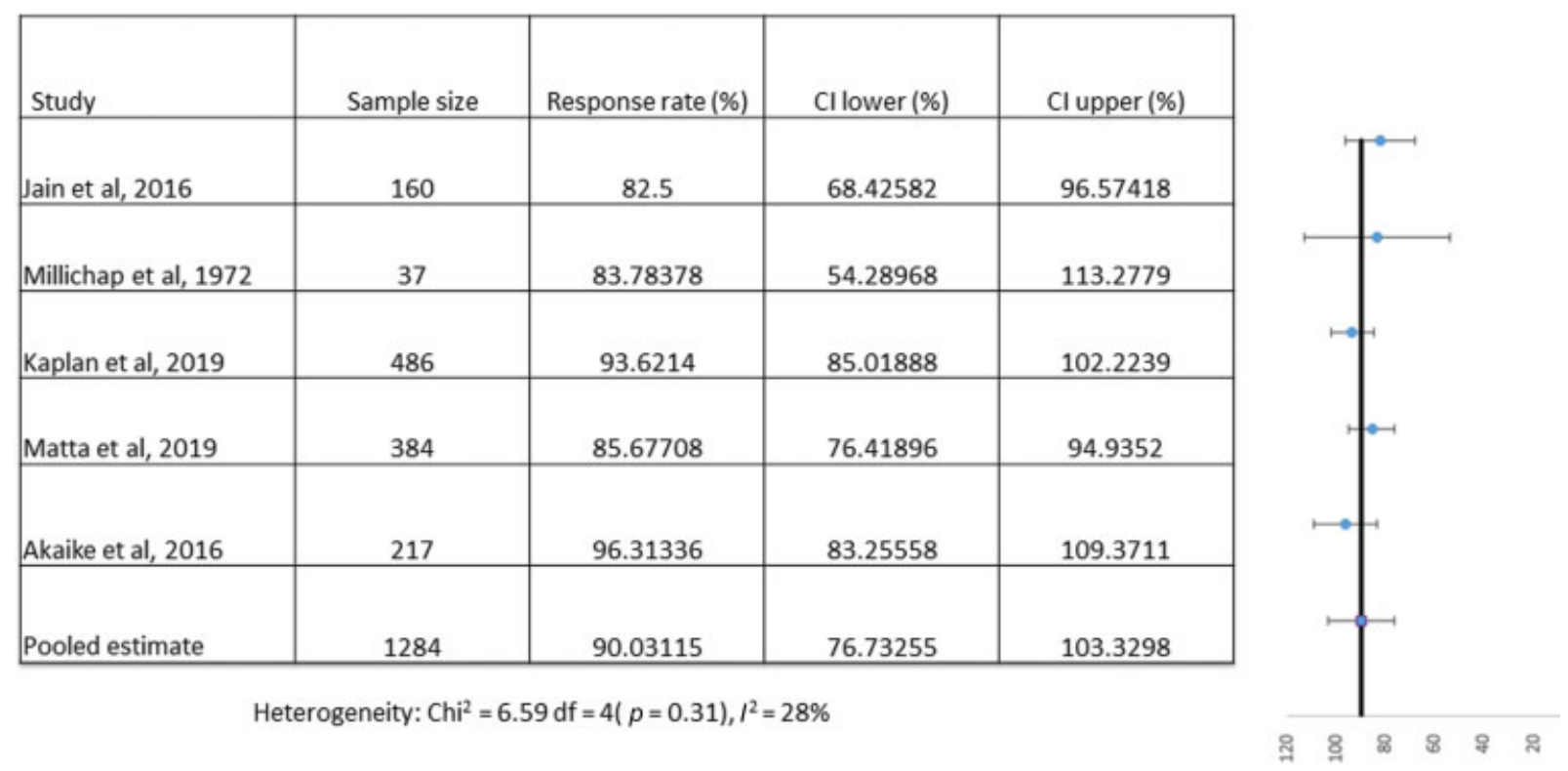

Fig. 4 Meta-analysis forest plot showing pooled estimate for the percentage of children who had a successful sleep EEG record after the first dose of triclofos. EEG, electroencephalogram.

medications appear similar in reported studies. ${ }^{19}$ Recently, some studies have been conducted in India, exploring its efficacy as a sedative in children and all these studies have shown good efficacy without any serious adverse effects. Consistent with this fact, in our review, most studies exploring triclofos were from Asia, while melatonin has been included in studies in different countries across the globe.

Our review could not answer the ideal sedative for children undergoing sleep EEG, as both of these drugs were found to have comparable efficacy. Although melatonin has an excellent safety profile, the success rate of only $76 \%$ with the first dose is not enough to consider it as an ideal sedative.
An ideal sedative for premedication for sleep EEG in children should be easy to administer, with a high success rate in achieving adequate sedation, acceptable sleep duration, short-sleep latency, and minimal adverse effects. Although none of the studies have explored the cost and availability issues of these two sedatives, both these medications in our review are easily available and affordable in most of the developed and even developing countries like India, with at least comparable prices in the Indian market. The yield of epileptiform abnormalities, although appears to be numerically higher with triclofos, but the difference is statistically insignificant. Taking into account all these factors, melatonin 
appears to be the reasonable first choice for this purpose followed by triclofos. However, as most of the studies in our review adapted their individual institute protocols, the role of personal preference of clinicians is also likely to play a significant role until international/national regulatory authorities come forward with specific guidelines or recommendations. The results of recently started RCT comparing these two medications in India is likely to add more substantial evidence in this regard. ${ }^{22}$ The direct head-to-head comparison studies between melatonin and chloral hydrate had conflicting results in the sense that Dirani et $\mathrm{al}^{14}$ found chloral hydrate to be more efficacious, whereas melatonin was found to be equally efficacious to chloral hydrate in the study by Ashrafi et al. ${ }^{5}$

We included the study comparing ramelteon and triclofos in the review, although the former is a selective melatonin receptor (MT1 and MT2) agonist and different from melatonin because this was the only study comparing directly the efficacy of ramelteon and triclofos. This direct comparison also yielded the same results as our systematic review such as both these medications had comparable efficacy and adverse effects were significantly more in number with triclofos. However, ramelteon has been described as a sleep-promoting medication without any direct sedating effect. It rather acts on melatonin receptors in the suprachiasmatic nucleus, affects the sleep regulatory mechanisms, and thereby enhances sleep. ${ }^{23}$ Existing literature suggests that ramelteon is 10 times more potent than melatonin, but in our review, the efficacy rate of ramelteon and melatonin in children was comparable. ${ }^{23}$ Studies comparing these two medications directly will provide more concrete information regarding the true efficacy of these two medications like the studies on chloral hydrate and triclofos.

In this review, we included results from children up to age 18 years, although most children aged 5 to 6 years or less mostly require sedatives and older children generally cooperate for obtaining a natural sleep record. However, in many instances, obtaining a natural sleep EEG record even for typically developed adolescents becomes difficult because of the alien environment of the neurophysiology laboratory, and children with developmental and behavioral abnormalities mostly require sedatives for the successful recording of sleep EEG. ${ }^{1}$

However, one aspect has not been explored by many studies utilizing melatonin that melatonin itself has some antiepileptic properties and may alter the EEG record like oral midazolam. ${ }^{24}$ By reducing glutamatergic and increasing gamma-aminobutyric acid (GABA) neurotransmission, it has been demonstrated that melatonin modulates the electrical activity of neurons. This indolamine is also metabolized to kynurenic acid in the body which is an endogenous anticonvulsant. In the recent literature, it has also been highlighted that melatonin has significant antiseizure properties. Melatonin has antiepileptic property which has been confirmed in experimental studies in mice where pentylenetetrazole and electrically induced seizures were conducted and it has been demonstrated that a significant increase in seizure threshold after administration of melatonin was reported. ${ }^{2}$

\section{Limitations}

Our systematic review has however several limitations which compromises the reliability of pooled estimate comparison between both the medications. The number of studies available with triclofos were few and the majority of them were retrospective studies. Studies comparing directly these two medications are yet to be completed. The included studies also did not adopt any uniform protocol for choosing the sedation drug and dose, thereby making the overall population of the review for any particular medication heterogeneous in many aspects. Most of the studies did not mention some of the outcome variables, so the pooled estimate for those variables was calculated from the available studies only. Especially for the variables, like adverse effects, sedation-related artifacts in EEG, and duration of sedation, few studies did not have mentioned the required information. The dose of melatonin varied across the studies and indirect comparison might have yielded some inconsistent results which are likely to change after completion of the currently undergoing head-to-head comparison trial. Lastly few studies attempted initially to obtain natural sleep records, and if unsuccessful, then progressed to administer these medications, while other studies straight away progressed to these sedatives. Still, this is the very first attempt at performing a comprehensive systematic review regarding the efficacy and safety profile of these two commonly used sedatives to record sleep EEG in children.

\section{Conclusion}

Evidence from existing literature, although weak, suggests the efficacy of triclofos and melatonin are comparable in inducing sleep for recording EEG in children. But triclofos is more likely to cause adverse effects. Further studies, especially RCTs, are required in this regard to generate more concrete evidence favoring any of these medications.

\section{Authors' Contributions}

All the authors contributed equally and share joint first authorship.

\section{Funding \\ None.}

\section{Conflict of Interest}

None declared.

\section{References}

1 Paasch V, Hoosier TM, Accardo J, Ewen JB, Slifer KJ. Technical tips: performing EEGs and polysomnograms on children with neurodevelopmental disabilities. Neurodiagn J 2012;52(04): 333-348

2 Aulakh R. Melatonin as an alternative to sedation in pediatric electroencephalography: need to exercise caution!. J Pediatr Neurol 2016;14:164-166

3 Wassmer E, Carter PF, Quinn E, et al. Melatonin is useful for recording sleep EEGs: a prospective audit of outcome. Dev Med Child Neurol 2001;43(11):735-738 
4 Loewy J, Hallan C, Friedman E, Martinez C. Sleep/sedation in children undergoing EEG testing: a comparison of chloral hydrate and music therapy. Am J Electroneurodiagn Technol 2006;46(04):343-355

5 Ashrafi MR, Mohammadi M, Tafarroji J, Shabanian R, Salamati P, Zamani GR. Melatonin versus chloral hydrate for recording sleep EEG. Eur J Paediatr Neurol 2010;14(03):235-238

6 Razieh F, Sharam J, Motahhareh G, Sedighah AK, MohammadHosein J. Efficacy of chloral hydrate and promethazine for sedation during electroencephalography in children; a randomised clinical trial. Iran J Pediatr 2013;23(01):27-31

7 Fallah R, Yadegari Y, Behdad S, Akhavan Karbasi S. Melatonin and intravenous midazolam administered orally in drug induced sleep electroencephalography of children: randomized clinical trial of efficacy. Arch Iran Med 2014;17(11):741-745

8 Sharawat IK, Panda PK, Kasinathan A, Panda P, Dawman L, Joshi K. Efficacy and tolerability of fenfluramine in patients with Dravet syndrome: a systematic review and meta-analysis. Seizure 2021; 85:119-126

9 Eisermann M, Kaminska A, Berdougo B, Brunet ML. Melatonin: experience in its use for recording sleep EEG in children and review of the literature. Neuropediatrics 2010;41(04):163-166

10 Ibekwe R, Jeaven L, Wilmshurst JM. The role of melatonin to attain electroencephalograms in children in a sub-Saharan African setting. Seizure 2017;51:87-94

11 Rizk T, Adote S, Vargas S, Camille C. Audit on: melatonin use in paediatric patients during neurophysiological procedures. J Neurosci Brain Imaging 2017;1(01):4-5

12 Gustafsson G, Broström A, Ulander M, Vrethem M, Svanborg E. Occurrence of epileptiform discharges and sleep during EEG recordings in children after melatonin intake versus sleep-deprivation. Clin Neurophysiol 2015;126(08):1493-1497

13 Alix JJP, Kandler RH, Pang C, Stavroulakis T, Catania S. Sleep deprivation and melatonin for inducing sleep in paediatric electroencephalography: a prospective multicentre service evaluation. Dev Med Child Neurol 2019;61(02):181-185

14 Dirani M, Nasreddine W, Melhem J, Arabi M, Beydoun A. Efficacy of the sequential administration of melatonin, hydroxyzine, and chloral hydrate for recording sleep EEGs in children. Clin EEG Neurosci 2017;48(01):41-47

15 Akaike H, Kondo E, Kono M, Kato A. Hypnotic effects of ramelteon during electroencephalography [in Japanese]. No To Hattatsu 2016;48(01):10-13

16 Jain P, Sharma S, Sharma A, Goel S, Jose A, Aneja S. Efficacy and safety of oral triclofos as sedative for children undergoing sleep electroencephalogram: an observational study. J Pediatr Neurosci 2016;11(02):105-108

17 Kaplan E, Daka A, Weissbach A, et al. Triclofos sodium for pediatric sedation in non-painful neurodiagnostic studies. Paediatr Drugs 2019;21(05):371-378

18 Matta GS, Peddisetty RP. Impact of etiology on efficacy of oral triclofos in recording pediatric electroencephalography: a tertiary care center study. J Neurosci Rural Pract 2019;10(02): 234-237

19 Millichap JG. Electroencephalographic evaluation of triclofos sodium sedation in children. Am J Dis Child 1972;124(04): 526-527

20 Wassmer E, Quinn E, Whitehouse W, Seri S. Melatonin as a sleep inductor for electroencephalogram recordings in children. Clin Neurophysiol 2001;112(04):683-685

21 Sander J, Shamdeen MG, Gottschling S, Gortner L, Gräber S, Meyer S. Melatonin does not influence sleep deprivation electroencephalogram recordings in children. Eur J Pediatr 2012;171(04): 675-679

22 Sleep inducing for EEG recording in children: a comparison between triclofos, melatonin, promethazine and hydroxyzine for sedation during EEG in children. Accessed July 1, 2021 at: https://www.cochranelibrary.com/central/doi/10.1002/central/ CN-01974494/full

23 Neubauer DN. A review of ramelteon in the treatment of sleep disorders. Neuropsychiatr Dis Treat 2008;4(01):69-79

24 Akyuz E, Kullu I, Arulsamy A, Shaikh MF. Melatonin as an antiepileptic molecule: therapeutic implications via neuroprotective and inflammatory mechanisms. ACS Chem Neurosci 2021; 12(08):1281-1292 